\title{
Medium modification of heavy meson spectra from QCD sum rules
}

\author{
Kei Suzuki* \\ Department of Physics, H-27, Tokyo Institute of Technology, Meguro, Tokyo 152-8551, Japan \\ E-mail: k.suzuki.2010@th.phys.titech.ac.jp \\ Philipp Gubler \\ RIKEN Nishina Center, RIKEN, Hirosawa 2-1, Wako, Saitama, 351-0198, Japan \\ E-mail: pgubler@riken.jp

\section{Makoto Oka} \\ Department of Physics, H-27, Tokyo Institute of Technology, Meguro, Tokyo 152-8551, Japan \\ E-mail: okadth.phys.titech.ac.jp
}

\begin{abstract}
Pseudoscalar $D$ meson spectral functions in nuclear medium are analyzed by employing QCD sum rules with the maximum entropy method. This approach enables us to extract the spectral functions without any phenomenological assumption, and thus to visualize modification of the spectral functions due to medium effects from nuclear medium. As a result, it is found that masses of both $D^{+}$and $D^{-}$mesons grow gradually with increasing density. Additionally, we construct charge conjugate projected sum rules and observed $D^{+}-D^{-}$mass splitting of about -20 $\mathrm{MeV}$ at nuclear saturation density.
\end{abstract}

XV International Conference on Hadron Spectroscopy-Hadron 2013

4-8 November 2013

Nara, Japan

\footnotetext{
*Speaker.
} 


\section{Introduction}

One of the most important problems in hadron physics is understanding the relation between chiral symmetry and hadron properties from Quantum chromodynamics (QCD) which describes the strong interaction. Hadrons in nuclear medium are known as a probe of chiral symmetry at finite density. For instance, $\rho, \omega$ and $\phi$ mesons in nuclear matter have been studied theoretically and experimentally for a long time. In the future, the CBM (Compressed Baryon Matter) experiment by FAIR (Facility for Antiproton and Ion Research) at GSI is expected to investigate the properties of open $(D, \bar{D})$ and hidden $\left(J / \psi, \eta_{c}\right)$ charmed mesons in hot and dense baryonic matter.

The QCD sum rule [1] is traditionally known as a powerful tool to investigate the properties of hadrons from QCD. Recently, it has become possible to apply the maximum entropy method (MEM) to QCD sum rules [2], which allows to extract the most probable form of the spectral function from the operator product expansion (OPE) of hadronic correlators without assuming some specific functional form, e.g. "pole + continuum" ansatz. This approach was shown to be successful in the $\rho$ meson [2], the nucleon with positive [3] or negative [4] parity sum rules in vacuum. Furthermore, it was applied to the spectral modifications at finite temperature for the charmonia [5] and bottomonia [6] channels.

Medium modification of $D$ mesons in nuclear matter has been investigated from various theoretical approaches. Among them, the only method directly based on QCD may be the QCD sum rule. More than ten years ago, Hayashigaki in [7] included only the condensates up to dimension 4, $\langle\bar{q} q\rangle,\left\langle\frac{\alpha_{s}}{\pi} G^{2}\right\rangle,\left\langle q^{\dagger} i \vec{D}_{0} q\right\rangle,\left\langle\frac{\alpha_{s}}{\pi}\left(\frac{(v G)^{2}}{v^{2}}-\frac{G^{2}}{4}\right)\right\rangle$ in the OPE, and observed that pseudoscalar $D$ meson mass shift is $-50 \mathrm{MeV}$ at nuclear saturation density $\rho_{0}$. Subsequently, Hilger et al. in [8] added the condensates up to dimension 5, $\langle\bar{q} g \sigma G q\rangle$, and $q_{0}$-odd terms, $\left\langle q^{\dagger} q\right\rangle,\left\langle q^{\dagger} \vec{D}_{0}^{2} q\right\rangle,\left\langle q^{\dagger} g \sigma G q\right\rangle$. As a result, they obtained a mass shift of $+45 \mathrm{MeV}$ at $\rho_{0}$ with serious ambiguity due to phenomenological density dependence of threshold parameter. In our approach, we apply MEM to QCD sum rules to obtain the results independently of such the threshold parameter. Also, Hilger et al. observed $D^{+}-D^{-}$mass splitting of $-60 \mathrm{MeV}$ at $\rho_{0}$. To improve this analysis, we introduce the charge conjugate projection as a novel approach.

\section{Method}

In the analyses of QCD sum rules, we start by defining the time-ordered hadronic current correlation function:

$$
\Pi^{J}(q)=i \int d^{4} x e^{i q \cdot x}\left\langle T\left[j^{J}(x) j^{J \dagger}(0)\right]\right\rangle,
$$

where $J$ labels the hadron species, $D^{+}, D^{-}, D^{0}$, and $\bar{D}^{0}$. In this work, we assume chiral limit $\left(m_{u}=m_{d}=0\right)$ and isospin symmetry $(\langle\bar{u} u\rangle=\langle\bar{d} d\rangle)$, so that $u$ and $d$ quarks are not distinguished. Then, we only need to exmine the pseudoscalar currents, $j^{D^{+}}=i \bar{d} \gamma_{5} c$ and $j^{D^{-}}=i \bar{c} \gamma_{5} d$. We also assume the spatial component of four momentum to be zero $: q=\left(q_{0}, \mathbf{0}\right)$. Contrary to the vacuum case in which the sum rule depends only on $q^{2}$ because of the Lorentz invariance, at finite density, we have to consider the terms of odd powers of $q_{0}$. Then, the correlators can be written in terms of the $q_{0}$-even and $q_{0}$-odd parts:

$$
\Pi^{J}\left(q_{0}\right)=\Pi^{\text {even }}\left(q_{0}^{2}\right)+q_{0} \Pi^{\text {odd }}\left(q_{0}^{2}\right)
$$


It is shown that $\Pi^{\text {even }}$ contains both the $D^{+}$and $D^{-}$spectra in both the positive and negative energy regions. Namely, for $\Pi^{D^{+}}$channel,

$$
\begin{aligned}
\Pi^{\text {even }}\left(q_{0}^{2}\right) & =\frac{1}{2}\left[\Pi^{+}\left(q_{0}^{2}\right)+\Pi^{-}\left(q_{0}^{2}\right)\right], \\
q_{0} \Pi^{\text {odd }}\left(q_{0}^{2}\right) & =\frac{1}{2}\left[\Pi^{+}\left(q_{0}^{2}\right)-\Pi^{-}\left(q_{0}^{2}\right)\right],
\end{aligned}
$$

where $\Pi^{+}\left(\Pi^{-}\right)$has $D^{+}\left(D^{-}\right)$spectrum in positive energy and $D^{-}\left(D^{+}\right)$spectrum in negative energy.

In order to separate $D^{+}$and $D^{-}$from $\Pi^{J}\left(q_{0}\right)$, we construct charge conjugate projected sum rule which is analogous to the parity projection for the baryon sum rules 94 . In this approach, we define the old-fashioned correlator, which includes only contribution in positive energy region:

$$
\Pi_{\mathrm{OPE}}^{D^{ \pm}} \equiv\left[\Pi^{ \pm}\left(q_{0}^{2}\right)\right]^{\text {old }}=\left[\Pi^{\text {even }}\left(q_{0}^{2}\right) \pm q_{0} \Pi^{\text {odd }}\left(q_{0}^{2}\right)\right]^{\text {old }} .
$$

Analyticity of the correlation functions is used to connect spectral functions to the imaginary part of OPE, and it is transformed by multiplying a kernel $W\left(q_{0}\right)$ as a weight function and we obtain the following integral sum rule:

$$
\begin{aligned}
\int_{-\infty}^{\infty} d q_{0} \frac{1}{\pi} \operatorname{Im} \Pi_{\mathrm{OPE}}^{D^{ \pm}} W\left(q_{0}, \hat{s}, \tau\right) & =G^{\mathrm{even}}(\hat{s}, \tau) \pm G^{\mathrm{odd}}(\hat{s}, \tau) \\
& =\int_{0}^{\infty} d \omega \rho^{ \pm}(\omega) W(\omega, \hat{s}, \tau) .
\end{aligned}
$$

In this study, we use the Gaussian kernel $W\left(q_{0}, \hat{s}, \tau\right)=\frac{1}{\sqrt{4 \pi \tau}} \exp \left[-\frac{\left(q_{0}^{2}-\hat{s}\right)^{2}}{4 \tau}\right]$. For the details of the $D$ meson OPE in nuclear medium, the readers are referred to [10]. To extract the spectral functions $\rho(\omega)$ from Eq. [2.6), we employ the MEM [2].

\section{Results}

Results of the $D$ meson spectral functions in vacuum and in nuclear medium are shown in Fig. 11and2 respectively. In vacuum, the $D^{+}$and $D^{-}$spectra are completely degenerate while they split at finite density. In the vacuum case, the first peak position is found at $1.78 \mathrm{GeV}$, which is consistent with the experimental value of the D meson ground state mass of $1.87 \mathrm{MeV}$. In nuclear medium, the peak residues are gradually suppressed as density increases while the peak positions are shifted to higher energy.

The detailed density dependence of the peak position is shown in Fig. 3 We note that the the $D^{-}$meson peak shift $\left(\sim 30 \mathrm{MeV}\right.$ at $\left.\rho_{0}\right)$ is stronger than that of the $D^{+}$meson $(\sim 10 \mathrm{MeV}$ at $\rho_{0}$ ). The mass difference between the $D^{+}$and the $D^{-}$is thus about $20 \mathrm{MeV}$ at $\rho_{0}$. This behavior is consistent with the picture that the $D^{-}$meson has a light quark which repulsively interacts with quarks in nuclear matter due to the Pauli blocking, so that the binding is weakened and the mass increases. On the other hand, the $D^{+}$meson has a light antiquark instead of a quark, so that it is not affected at least by the Pauli blocking. From the viewpoint of QCD sum rule, the $D^{+}-D^{-}$ mass splitting is caused by the sign of $q_{0}$-odd terms, $\left\langle q^{\dagger} q\right\rangle,\left\langle q^{\dagger} \vec{D}_{0}^{2} q\right\rangle,\left\langle q^{\dagger} g \sigma G q\right\rangle$ in Eq. (2.5). These results qualitatively agree with Ref. [8] which employs a "pole + continuum" ansatz. 


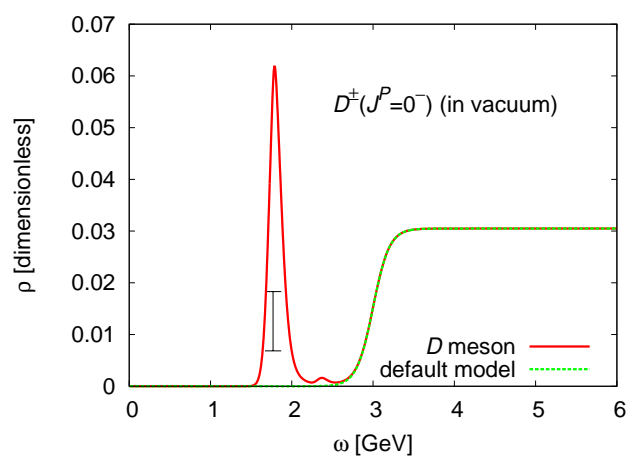

Figure 1: Spectral function extracted from $D^{ \pm}$meson sum rule in vacuum with MEM. Definition of the error bar is given in [2]
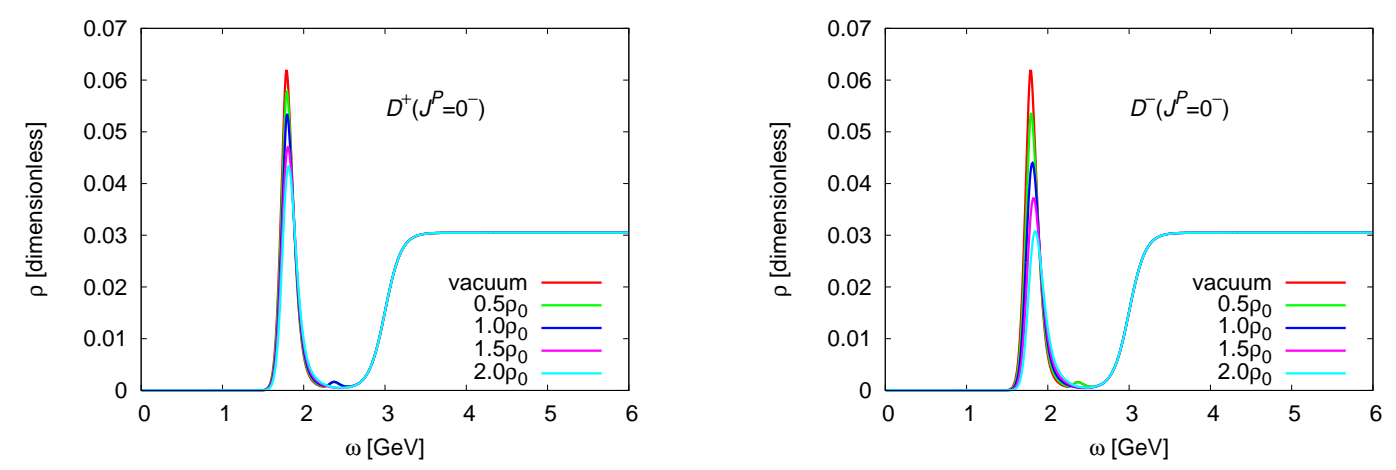

Figure 2: Spectral functions extracted from $D^{ \pm}$meson sum rules in nuclear medium with MEM. $\rho_{0}$ is the nuclear saturation density.

\section{Conclusion and Outlook}

In summary, we have investigated the pseudoscalar $D$ meson in nuclear medium by using QCD sum rules and MEM. In order to separate $D^{+}$and $D^{-}$into independent peaks, we have constructed charge conjugate projected sum rules. As a result, we found that both $D^{+}$and $D^{-}$peaks are shifted to higher energy with increasing density. Also, we observed a $D^{+}-D^{-}$mass splitting of about -20 $\mathrm{MeV}$ at $\rho_{0}$. As a next topic, we will analyze $B^{+}$and $B^{-}$mesons in nuclear matter. Furthermore, it would be interesting to systematically examine the heavy quark mass $\left(m_{h}\right)$ dependence of the behavior of heavy-light hadrons in nuclear medium, comprehensively expanding from the heavy quark limit $\left(m_{h} \rightarrow \infty\right)$ to the chiral limit $\left(m_{h} \rightarrow 0\right)$.

\section{References}

[1] M.A. Shifman, A.I. Vainshtein, and V.I. Zakharov, Nucl. Phys. B147, 385 (1979); B147, 448 (1979). 


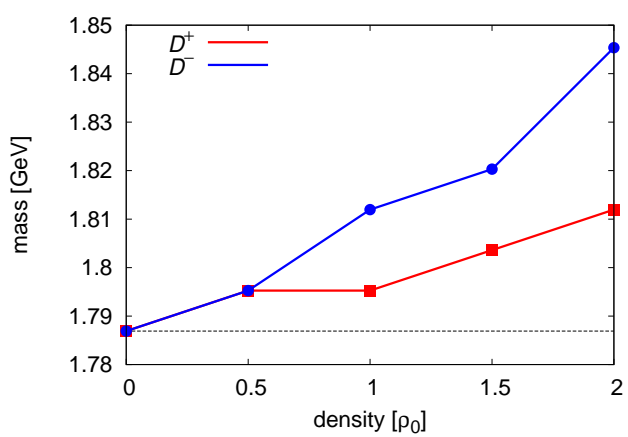

Figure 3: Density dependence of peak positions in $D^{ \pm}$spectra. Dotted line shows peak position in vacuum.

[2] P. Gubler and M. Oka, Prog. Theor. Phys. 124, 995 (2010) [arXiv:1005.2459 [hep-ph]].

[3] K. Ohtani, P. Gubler and M. Oka, Eur. Phys. J. A 47, 114 (2011) [arXiv:1104.5577 [hep-ph]].

[4] K. Ohtani, P. Gubler and M. Oka, Phys. Rev. D 87, 034027 (2013) [arXiv:1209.1463 [hep-ph]].

[5] P. Gubler, K. Morita and M. Oka, Phys. Rev. Lett. 107, 092003 (2011) [arXiv:1104.4436 [hep-ph]].

[6] K. Suzuki, P. Gubler, K. Morita and M. Oka, Nucl. Phys. A897, 28 (2013) [arXiv:1204.1173 [hep-ph]].

[7] A. Hayashigaki, Phys. Lett. B487 96 (2000) [arXiv:nucl-th/0001051].

[8] T. Hilger, R. Thomas and B. Kämpfer, Phys. Rev. C 79025202 (2009) [arXiv:0809.4996 [nucl-th]].

[9] D. Jido, N. Kodama and M. Oka, Phys. Rev. D 544532 (1996) [arXiv:hep-ph/9604280].

[10] S. Zschocke T. Hilger and B. Kämpfer, Eur. Phys. J. A 47, 151 (2011) [arXiv:1112.2477 [hep-ph]]. 